\title{
Local Generation of Fast Ripples in Epileptic Brain
}

\author{
Anatol Bragin,, ${ }^{1,3}$ Istvan Mody, ${ }^{1,3}$ Charles L. Wilson, ${ }^{1,3}$ and Jerome Engel Jr' ${ }^{1,2,3}$ \\ Departments of ${ }^{1}$ Neurology and ${ }^{2}$ Neurobiology and the ${ }^{3}$ Brain Research Institute, University of California at Los Angeles \\ School of Medicine, Los Angeles, California 90095
}

Aperiodic high-frequency oscillations $(>100 \mathrm{~Hz}$ ) reflect a shortterm synchronization of neuronal electrical activity. It has been shown in the epileptic brain that spontaneous oscillations in the frequency range of $250-600 \mathrm{~Hz}$ reflect action potential population bursts of synchronously discharging neuronal clusters. These oscillations occur in the early stages of epileptogenesis in areas adjacent to the brain lesion and may trigger the formation of seizure-generating neuronal networks. We studied the extent of the area generating oscillations in the frequency range of 250-600 Hz [fast ripples (FRs)] in intrahippocampal kainic acid-treated rats with spontaneous seizures, by analyzing voltage versus depth profiles of FRs in hippocampal and parahippocampal areas in freely moving animals and by spatial map- ping in hippocampal slice preparations in vitro. The strength of inhibition was compared in areas with and without FRs using a paired-pulse paradigm. The extent of the areas generating FRs did not exceed $1 \mathrm{~mm}^{3}$. The areas generating FRs became broader after the application of the $\mathrm{GABA}_{\mathrm{A}}$ receptor antagonist bicuculline. Paired-pulse fast inhibition at 15-30 msec intervals was similar in areas generating FRs and areas not generating FRs. Our data illustrate that hypothesized clusters of highly interconnected neurons are capable of overcoming interneuron feedback inhibition, resulting in generation of epileptiform bursts, eventually leading to seizure activity.

Key words: hippocampus; entorhinal cortex; chronic seizures; fast ripples; kainic acid; oscillation
High-frequency oscillations in the frequency range of 100-600 $\mathrm{Hz}$ indicate a short-term synchronization of neuronal ensembles (Buzsaki et al., 1992; Curio, 2000; Jones et al., 2000; Grenier et al., 2001). In primary sensory areas, these oscillations occur during the acquisition of sensory information, whereas in the hippocampal-entorhinal circuitry, they occur during immobility and/or slow wave sleep. In sensory systems, the high-frequency oscillations reflect coherent discharges of neurons in response to incoming sensory events (Curio, 2000; Jones et al., 2000) and are generated as a result of sequential and recurrent propagation of action potentials throughout the principal cell population (Kandel and Buzsaki, 1997; Jones et al., 2000). Two mechanisms of high-frequency oscillation generation have been proposed in the hippocampus. Draguhn et al. (1998) suggested that axoaxonal gap junctions are involved in the generation of high-frequency bursts in vitro. Ylinen et al. (1995) presented evidence that highfrequency oscillations (ripples) in vivo reflect fast IPSPs on the somata of pyramidal cells.

In the hippocampus and entorhinal cortex of rats with recurrent spontaneous seizures, we described recently (Bragin et al., 1999a) high-frequency $(250-500 \mathrm{~Hz})$ oscillations that were termed fast ripples (FRs), because they are faster than the normal ripple oscillations (100-200 $\mathrm{Hz})$ recorded from those structures (Buzsaki et al., 1992). They appear to reflect field events composed of hypersynchronous action potentials and may be an EEG analog of epileptiform events consisting of bursts of population spikes that have been described previously in many in vivo and in

Received Oct. 22, 2001; revised Dec. 14, 2001; accepted Dec. 19, 2001.

This work was supported by National Institutes of Health Grants NS-33310 and NS-02808. We thank Joyel Almajano for essential assistance in the experiments and Dr. G. W. Mathern for useful discussion of histological data.

Correspondence should be addressed to Dr. Anatol Bragin, 2147 RNRC, Department of Neurology, University of California at Los Angeles School of Medicine, 710 Westwood Plaza, Los Angeles, CA 90095-1769. E-mail: abragin@ucla.edu. Copyright (C) 2002 Society for Neuroscience $0270-6474 / 02 / 222012-10 \$ 15.00 / 0$ vitro acute models of epilepsy. Similar patterns of high-frequency oscillations have been observed in the epileptic zones of patients with temporal lobe epilepsy (Bragin et al., 1999b), and several studies have shown that sometimes focal seizures begin with high-frequency oscillations (Fisher et al., 1992; Bragin et al., 1999b; Traub et al., 2001).

In rat models of chronic epilepsy, FRs are seen during the latent period between the occurrence of an initial epileptogenic insult and the onset of recurrent spontaneous seizures (Bragin et al., 2000). It was hypothesized that FRs reflect the activity of pathologically interconnected clusters of neurons (PIN clusters) and play an important role in epileptogenesis, acting as a trigger for pathological synaptic modifications in target brain areas. The consequence of abnormal synaptic modifications is the formation of a distributed network of PIN clusters, which may be a functional substrate for epilepsy (Bertram et al., 1998; Bragin et al., 2000).

In the present experiments, the properties of fast ripples were compared in kainic acid (KA)-treated rats in both in vivo and in vitro experiments. Our specific objective was to determine the extent of the area generating FRs using voltage versus depth profiles derived from in vivo experiments and sequential spatial mapping derived from in vitro experiments. A second objective was to compare the strength of inhibition in areas generating FRs and those not generating FRs. A third objective was to analyze the interaction between areas generating FRs in the dentate gyrus, subiculum, and entorhinal cortex of freely moving rats.

\section{MATERIALS AND METHODS}

All procedures described in this study were approved by the University of California, Los Angeles, Institutional Animal Care and Use Committee. Twenty-four rats with recurrent spontaneous seizures and seven naive age-matched control rats were used for the experiments.

Kainic acid injection. Adult male (250-350 gm) Sprague Dawley rats were given atropine ( $0.04 \mathrm{mg}$, i.m.), anesthetized with chloral hydrate $(400 \mathrm{mg} / \mathrm{kg}$, i.p. $)$, and unilaterally injected with $\mathrm{KA}(0.4 \mu \mathrm{g} / 0.2 \mu \mathrm{l}$ normal 
saline) in the right posterior hippocampus [anteroposterior (AP), -5.6 $\mathrm{mm}$; mediolateral (ML), $4.0 \mathrm{~mm}$; dorsoventral (DV), $7.0 \mathrm{~mm}$ ]. Beginning 3-4 months after injection, rats were observed during repeated $16-24 \mathrm{hr}$ video monitoring periods for 1-2 weeks every month to detect spontaneous behavioral seizures.

Microelectrode implantation. After completion of preliminary video monitoring, rats demonstrating spontaneous seizures were anesthetized with a mixture of ketamine $(100 \mathrm{mg} / \mathrm{kg})$, xylazine $(5.2 \mathrm{mg} / \mathrm{kg})$, and acepromazine $(1.0 \mathrm{mg} / \mathrm{kg})$, administered intramuscularly.

Pairs of tungsten wires $(60 \mu \mathrm{m}$ in diameter) with $0.5 \mathrm{~mm}$ vertical tip separation were placed in the right angular bundle to stimulate perforant path afferents to the hippocampus (AP, $-7.0 \mathrm{~mm}$ from bregma; ML, 3.5 $\mathrm{mm}$; and DV, $2.5 \mathrm{~mm}$ from the surface of neocortex). Fixed recording microelectrodes also consisted of pairs of tungsten wires with $1.5 \mathrm{~mm}$ vertical tip separation. They were implanted bilaterally at symmetrical points in the dentate gyrus (DG) and CA1 regions of anterior hippocampus (AP, $-3.5 \mathrm{~mm}$; ML, $2.0 \mathrm{~mm}$; DV , 3.5-4.5 mm), the DG region of the posterior hippocampus (AP, $-5.6 \mathrm{~mm}$; ML, $4.0 \mathrm{~mm}$; DV, $5.0 \mathrm{~mm}$ ), and entorhinal cortex (EC) (AP, - $8.0 \mathrm{~mm}$; ML, $5.0 \mathrm{~mm}$; DV, $7.0 \mathrm{~mm}$ )

An array of four microelectrodes, fixed in relation to one another but moveable together in the vertical plane (tungsten, $60 \mu \mathrm{m}$ in diameter, insulated except at the tips), was used for hippocampal recordings. The wires were arrayed in a single plane, with each wire separated horizontally from the adjacent wire by $300 \mu \mathrm{m}$. Vertically, the tips of each successive wire were $500 \mu \mathrm{m}$ longer than the adjacent wire, achieving separation both horizontally and vertically. During initial implantation, the electrode tips were oriented along the long axis of the hippocampus and lowered vertically into the brain to a point just above the dorsal hippocampus. Arrays were implanted in one or both mid-dorsal hippocampi $[\mathrm{AP},-3.0 \mathrm{~mm}$; lateral $(\mathrm{L}), 2.6 \mathrm{~mm}$ ] and/or more posteriorly (AP, $-5.6 \mathrm{~mm}$; $\mathrm{L}, 4.0 \mathrm{~mm}$ ). The locations of the recording electrodes in the DG were chosen on the basis of the shape of the evoked potentials elicited by perforant path stimulation. Electrodes with responses consisting of a positive field EPSP (fEPSP) with a superimposed negative population spike of 3-8 msec latency were considered to be located near the granule cell layer of the DG.

Data acquisition. During in vivo recordings in freely moving rats, five four-channel MOSFET input operational amplifiers mounted in the cable connector served to eliminate cable movement artifacts. Physiological data were recorded wide band $0.1 \mathrm{~Hz}$ to $5.0 \mathrm{kHz}$ and sampled at 10 $\mathrm{kHz} /$ channel (16 channels) with 12 -bit precision on a Pentium personal computer using RC Electronics (Santa Barbara, CA) software. The data were stored on JAZ cartridges and archived on compact disks. To measure depth profiles of the evoked and spontaneous field potential, electrical activity was recorded every $100 \mu \mathrm{m}$ as the electrodes were advanced down through the hippocampus. Paired-pulse testing was used during stimulation of perforant path and recording of DG responses to determine the strength of paired-pulse depression. Stimulus pairs (pulse duration, $0.1 \mathrm{msec}$; current, $0.1-0.5 \mathrm{~mA}$ ) were separated by $10 \mathrm{sec}$ and were given at interstimulus intervals of $15-1000 \mathrm{msec}$.

Histological procedures. At the end of the electrophysiological experiments, rats were deeply anesthetized and perfused with $2.5 \%$ paraformaldehyde before Nissl and neo-Timm's staining to verify electrode placements and evaluate mossy fiber sprouting in the dentate gyrus (Mathern et al., 1995).

In vitro studies. Three KA-treated rats with documented spontaneous seizures and seven control rats were used for in vitro experiments. Rats were deeply anesthetized with $50 \mathrm{mg} / \mathrm{kg}$ Nembutal, the brains were removed, and horizontal $400-\mu \mathrm{m}$-thick slices containing the EC, subiculum, and hippocampal formation in the horizontal plane were prepared using a Lancer Series 1000 vibratome. The brains were cooled for $\geq 1$ min in $4^{\circ} \mathrm{C}$ artificial CSF (ACSF) containing (in $\mathrm{mM}$ ): $126 \mathrm{NaCl}, 1.25$ $\mathrm{NaH}_{2} \mathrm{PO}_{4}, 26 \mathrm{NaHCO}_{3}, 2.5 \mathrm{KCl}, 2 \mathrm{CaCl}_{2}, 2 \mathrm{MgCl}_{2}$, and $10 \mathrm{D}$-glucose, $\mathrm{pH}$ 7.4. During sectioning, slices were sequentially placed in the incubation chamber to determine the location of slices adjacent to the KA lesion during subsequent electrophysiological testing. Before recordings, the slices were incubated at $32^{\circ} \mathrm{C}$ for $1-6 \mathrm{hr}$ in a $300 \mathrm{ml}$ storage chamber filled with ACSF and continuously bubbled with a mixture of $95 \%$ $\mathrm{O}_{2}-5 \% \mathrm{CO}_{2}$. In experiments requiring a decreased level of $\mathrm{GABA}_{\mathrm{A}}$ receptor-mediated inhibition, slices were perfused with $10 \mu \mathrm{M}$ bicuculline (Sigma, St. Louis, MO) in ACSF containing $5 \mathrm{~mm} \mathrm{KCl}$.

Electrical activity was recorded using standard, extracellular, ACSFfilled glass microelectrodes $(\sim 10 \mathrm{M} \Omega$ impedance $)$ in the DG granular cell layer, CA1, and subicular areas of hippocampus. The stimulating electrodes were placed in the perforant path and hilus in the DG experiments and in stratum radiatum and alveus in the CA1 slice experiments. Rectangular pulses of $0.1 \mathrm{msec}$ duration with currents ranging from 0.1 to $0.5 \mathrm{~mA}$ were used for electrical stimulation. Amplified signals were low-pass filtered at $3 \mathrm{kHz}$, sampled at $10 \mathrm{kHz}$, and collected using a National Instruments (Austin, TX) analog-to-digital interface.

After completion of the electrophysiological experiments, slices placed between two coverslips were incubated in $0.1 \% \mathrm{Na}_{2} \mathrm{~S}$ for $10 \mathrm{~min}$ and were transferred to a $2.5 \%$ paraformaldehyde solution for 2-4 d. Slices were then cut with a vibratome into $50 \mu \mathrm{m}$ sections and stained with Nissl or neo-Timm's methods (Mathern et al., 1995).

Data analysis was performed off-line on a Pentium computer, using RC Electronics software. For in vivo studies, videotapes were reviewed, and detected seizures were scored on the basis of Racine's scale (Racine, 1972). The degree of paired-pulse suppression was measured as a ratio of the test pulse response to the conditioning pulse response. The amplitude and number of waves within high-frequency oscillations or number of population spikes in a burst was counted only if they met a 3:1 signal/ background noise criterion. An all-point amplitude histogram was computed from activity recorded in the absence of FR events, and background noise was defined as the peak amplitude of the amplitude histogram. Averages of spontaneous oscillatory or population events were obtained by setting a detection level that triggered peaks of the bandpass filtered field activity at least three times greater than background. The lower limit of the digital bandpass filter (roll-off of $36 \mathrm{~dB}$ ) was arbitrarily set at $175 \mathrm{~Hz}$ to exclude any low-frequency population events and normal ripple activity, which primarily ranges from 100 to 200 $\mathrm{Hz}$. The upper limit of the bandpass filter was set at $600 \mathrm{~Hz}$ to eliminate any contribution of extracellularly recorded action potentials to the triggered averages.

Individual power spectra were calculated in each animal from no less than 50 oscillations. These averaged power spectra were again averaged across the animals, picking the inter-animal variation of the FR power spectra.

The position of the microelectrode during voltage versus depth profiles was localized based on responses to perforant path stimulation. Responses with a positive fEPSP and negative population spike were considered to be recorded in the inner molecular layer of the DG just above the granular layer (for details, see Lomo, 1971; Bragin et al., 1995) (see Figure 2, record 2 in Results). This point was taken as the zero point in histological analysis of the section containing the microelectrode track. The absence of the population spike in the evoked potential was considered evidence of passing through the upper blade of the granular layer, and the reoccurrence of the population spike was considered evidence of recording from the lower blade of the granular layer (see Fig. 2, records $2,17)$. The distance between this point and the zero point was calculated by multiplying the number of steps by the length of the step $(100 \mu \mathrm{m})$ to estimate the calibration for location of each recording point within the track. The ratio between the estimated size of the brain section during photographing and the size of the section estimated on the basis of the distance between blades of the granule layers during electrophysiological experiments indicated the percentage of tissue shrinkage during histological study.

Data were analyzed using ANOVA and appropriate individual group tests of significance. Data are expressed as means \pm SD, and statistical comparisons were made by applying Student's $t$ test. The significance level was set at $p<0.05$.

\section{RESULTS}

In 15 of 24 freely moving epileptic rats, electrical activity was recorded with fixed microelectrodes. In six rats, movable microelectrodes were used, and, in three of the six animals, voltage versus depth profiles of the evoked and spontaneous FRs were analyzed. Three epileptic rats (13 slices) and seven naive control rats (26 slices) were used for in vitro slice preparation experiments.

\section{Comparison of FR properties in vivo and in vitro Spontaneous fast ripples in vivo}

In agreement with our previous study, in freely moving epileptic rats (Bragin et al., 1999), spontaneous FRs were found in the DG and CA1 areas of the posterior hippocampus adjacent to the KA lesion site, as well as in the ipsilateral EC. Of 15 rats with 

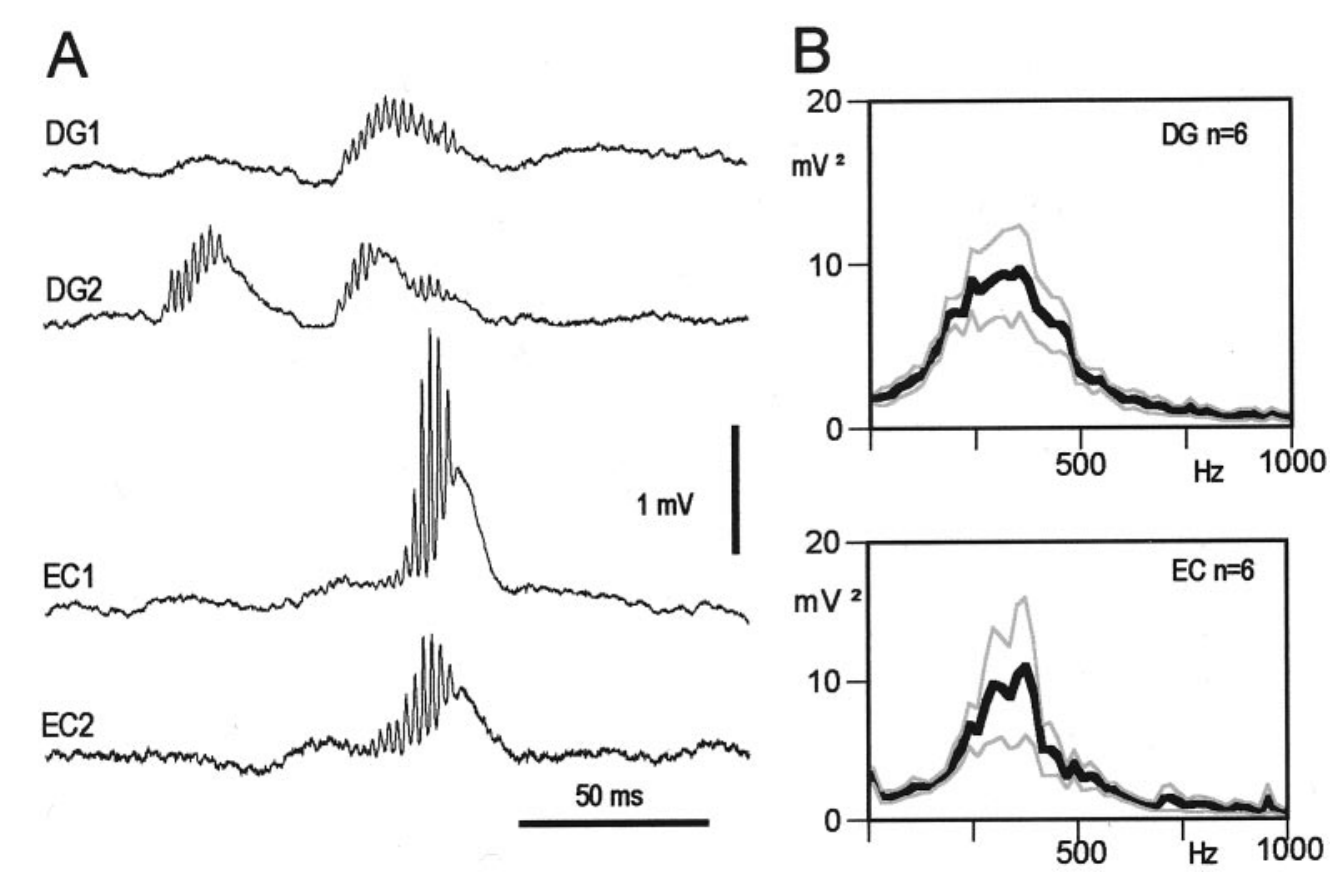

Figure 1. A, An example of FRs recorded simultaneously in the dentate gyrus and entorhinal cortex of an epileptic rat 4 months after kainic acid injection in the CA3 area of the posterior hippocampus. $B$, Averaged power spectra of FRs in the DG (top) and EC (bottom) of six rats (in square millivolts, here and in other figures). Gray lines illustrate SEM.

implanted fixed microelectrodes, FRs were found in nine. FRs were observed in all six rats with movable microelectrodes. In Figure $1 A$, while recording with pairs of fixed microelectrodes separated vertically by $1.5 \mathrm{~mm}$ in DG and EC areas, from one moment to the next, FRs could be recorded by both microelectrodes, or locally, by one or the other of the two electrode tips. Note in Figure $1 A$, for example, that first FR occurs in DG2 but is absent in DG1, EC1, and EC2. In all cases within the EC, FRs occurred simultaneously on both microelectrodes spaced $1.5 \mathrm{~mm}$ apart. The frequency of FR oscillation ranged between 200 and $650 \mathrm{~Hz}$. Power spectral analysis (Fig. 1B) illustrates that FR frequency in the DG of different animals ranged between 200 and $450 \mathrm{~Hz}$, with the peak at $370 \mathrm{~Hz}$. In the EC, the frequency range of FRs was between 200 and $400 \mathrm{~Hz}$, with the peak at $375 \mathrm{~Hz}$. In rats with fixed microelectrodes, FRs were recorded from the same microelectrodes for a period of 2-3 months.

The maximum FR amplitude was measured in six rats with movable recording microelectrodes. The range of these maximal FR amplitudes of six rats was between 0.1 and $0.85 \mathrm{mV}$ in the DG and between 0.1 and $1.5 \mathrm{mV}$ in the EC.

\section{Stimulation-evoked fast ripples in vivo}

In areas of FR generation in the DG and CA1 regions of the hippocampus, responses to perforant path stimulation contained a population spike followed by FRs with latency of 10-12 msec. In the entorhinal cortex in areas of spontaneous FR generation, FRs were evoked by antidromic perforant path stimulation, with 3-7 msec latency. Mapping of perforant path evoked and spontaneous FRs in the DG at $100 \mu \mathrm{m}$ increments showed that FRs were generated in local areas with a cross section of $\sim 1 \mathrm{~mm}$. An example of voltage versus depth profile mapping of DG-evoked potentials in response to perforant path stimulation is presented in Figure $2 A$. It shows that FRs occurred only at recording points 18 and 19 , as well as $500 \mu \mathrm{m}$ deeper at point 24. During stimulation protocols of five shocks per recording site, averaged responses such as those shown in Figure $2 A$ occurred with a probability of $80 \%$ at sites that showed a response and of $0 \%$ at sites that showed no responses $(n=6$ rats). At these same
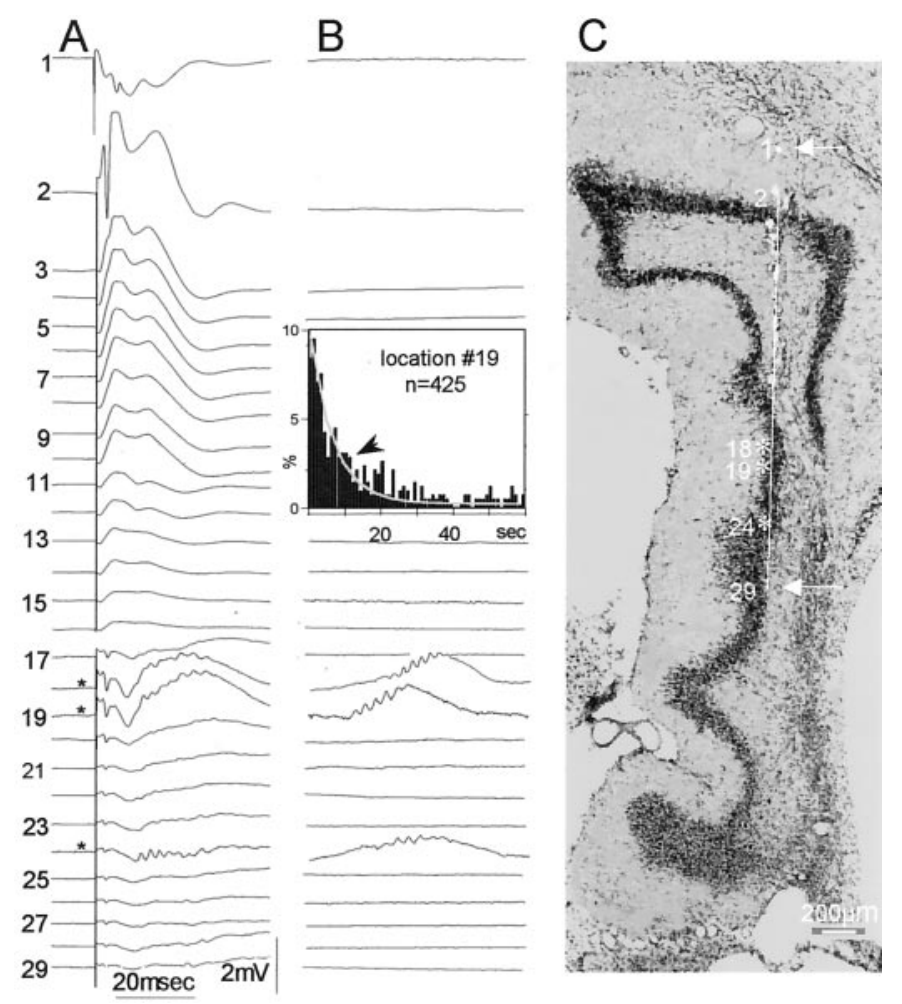

Figure 2. A, Voltage-depth profiles of potentials recorded at $100 \mu \mathrm{m}$ intervals in response to perforant path stimulation. Note that FRs are evoked only at sites 18,19 , and 24 . Recordings are averages of five stimulations at each side. $B$, Spontaneous FRs could be recorded only at the same sites as in $A: 18,19$, and 24 (average of 5 in each trial). Inset illustrates distribution of spontaneous FRs $(n=425$ FR events) recorded at location 19 during a period of $120 \mathrm{~min}$. Arrow indicates mean inter-FR interval. $C$, Track of the microelectrode in a Nissl-stained coronal section of the posterior curve of the hippocampus, showing the dorsoventral extent of the dentate gyrus. Arrows indicate the beginning and the end the recording area. Stars indicate sites at which FRs were recorded. Scale bar: gray, 200 $\mu \mathrm{m}$ on the basis of histological measurement; white, $200 \mu \mathrm{m}$ on the basis of electrophysiological measurement (for details, see Materials and Methods). 


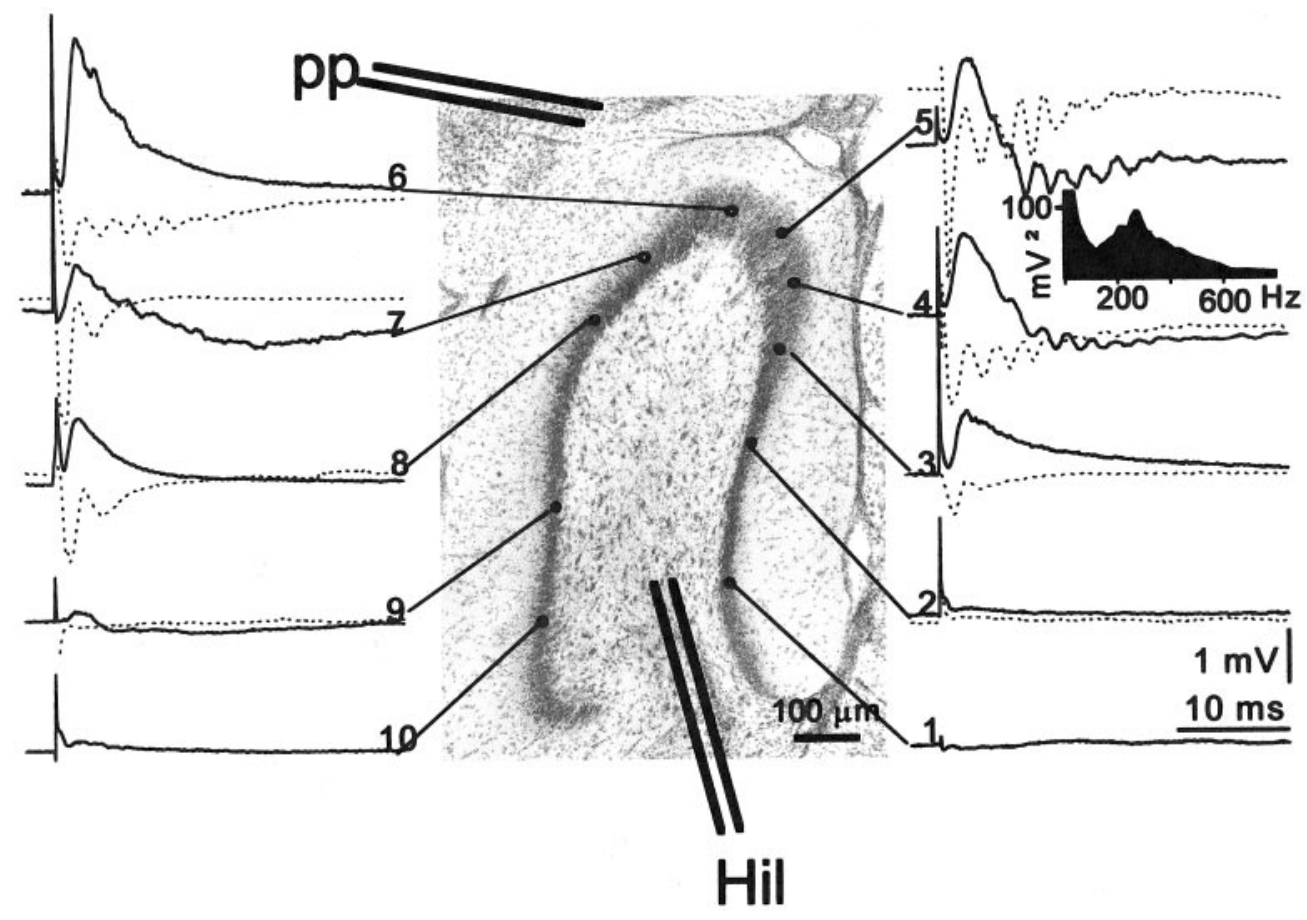

Figure 3. Map of evoked field potentials in the dentate gyrus slices obtained from an epileptic rat in response to perforant path (solid lines) and hilus (dashed lines) stimulation. Notice that FR-like responses are recorded at points 4,5 , and 6 in response to both perforant path and hilar stimulation. The inset is the power spectra of the evoked responses to hilus stimulation. Each recording is the average of five responses in this and remaining figures. recording points, FRs were also found to occur spontaneously (Fig. 2B). Each recording site was monitored for 3-5 min without stimulation to determine whether spontaneous FRs were present, but the only successful detection of FRs occurred at sites 18, 19, and 24. At these sites, while recording from the animal illustrated in Figure $2 B$, several hundred spontaneous oscillations were recorded over a period of several hours, and the averages of these oscillations are shown in the figure. The frequency distribution of inter-FR intervals in the inset of Figure 2 is a single exponential and is consistent with their random occurrence. The inset also shows that inter-FR intervals were often as long as $1 \mathrm{~min}$ but that the median interval was $9 \mathrm{sec}$ in a total of nine animals. In Figure $2 C$, part of the microelectrode track from which the recordings in $A$ and $B$ were made is shown, indicating the anatomical recording points generating FRs were adjacent to and within the granule cell layer of the dentate gyrus.

\section{Stimulation-evoked fast ripples in vitro}

In six of 13 slices in normal medium, the responses to electrical stimulation of afferents consisted of high-frequency (200-600 $\mathrm{Hz}$ ) oscillations resembling a burst of population spikes. Because of the similarity of these responses to the in vivo responses recorded in areas generating spontaneous FR, we will call them "FR-like" responses. All six slices in which FR-like responses were recorded were from areas of hippocampus adjacent to the KA lesion, and no slices showing FR-like activity were located $>3.0 \mathrm{~mm}$ from the lesion $(n=7)$. None of the control slices $(n=$ 26) showed FR-like activity.

Mapping of DG-evoked field potentials in response to perforant path stimulation showed that FR-like responses were found only in restricted areas. Outside of these limited areas, responses consisted only of an fEPSP with a single population spike or without a population spike (Fig. 3). The same areas that responded to perforant path stimulation with an FR-like response also responded to hilus stimulation with an FR-like response (Fig. 3, dashed lines).

In the CA1 region of the hippocampus, FR-like responses also showed a limited spatial distribution (Fig. 4). CA1 areas that responded with FR-like responses during stimulation of the Schaffer collaterals also responded with FR-like responses to alvear stimulation. In parahippocampal regions, localized areas also responded to electrical stimulation with an FR-like pattern. An example of such a response is presented in Figure 5, in which the areas labeled $6 a$ and $6 b$ located in the deep layers of subiculum responded with FR-like responses to electrical stimulation of the subiculum itself or of the parasubiculum.

\section{Inhibition in areas generating and not generating FRs Stimulation-evoked fast ripples in vivo}

The strength of inhibition in areas generating FRs and those not generating FRs was compared using the paired-pulse test. During in vivo experiments, the conditioning stimulus pulse current was adjusted to evoke a clear population spike in the DG granular layer, as shown in Figure $6 A$ (dashed line). With paired stimulation at $30 \mathrm{msec}$ interpulse intervals, the initial population spike response to the test pulse was completely suppressed, regardless of whether or not the area could generate FRs (Fig. 6B). At 50-500 msec interpulse intervals, in areas not generating FRs (noFRPS), the test pulse-evoked population spike was partially suppressed, whereas in areas generating FRs (FRPS), the test pulse-evoked population spike became augmented by $120-150 \%$ at $80-200 \mathrm{msec}$ interpulse intervals. It was noted during pairedpulse testing that changes in the population spike were accompanied by significant changes in the late components of the field response. Specifically, as shown in Figure $6 A$ (solid line) with a paired-pulse interval of $500 \mathrm{msec}$, an FR-like discharge occurred with a latency of $20 \mathrm{msec}$ only in response to the test pulse. In Figure $6 C$, the FR-like event was augmented from 340 to $530 \%$ in response to the test pulse at interpulse intervals ranging from 70 to $500 \mathrm{msec}$. In comparison, there was no significant change in the late components at those interpulse intervals in areas not generating FRs (data not shown). 
Figure 4. Map of evoked potentials in the CA1 area of the hippocampus of an epileptic rat in response to alveus (Stim alv) and stratum radiatum (Stim $\mathrm{rad}$ ) stimulation. Notice that FR-like responses occurred at recording point 3 in response to both points of stimulation. The inset is the power spectrum of the response in area 3 to alveus stimulation.
Figure 5. Map of evoked potentials showing FR-like responses in the deep layer of the subiculum-presubiculum area of an epileptic rat in response to deep layer stimulation. Notice that fast ripples were evoked in the deep layer of the subiculum in response to stimulation of both subicular and parasubicular electrodes. The inset is the power spectrum of the evoked response to the st 1 stimulation at point 6 .
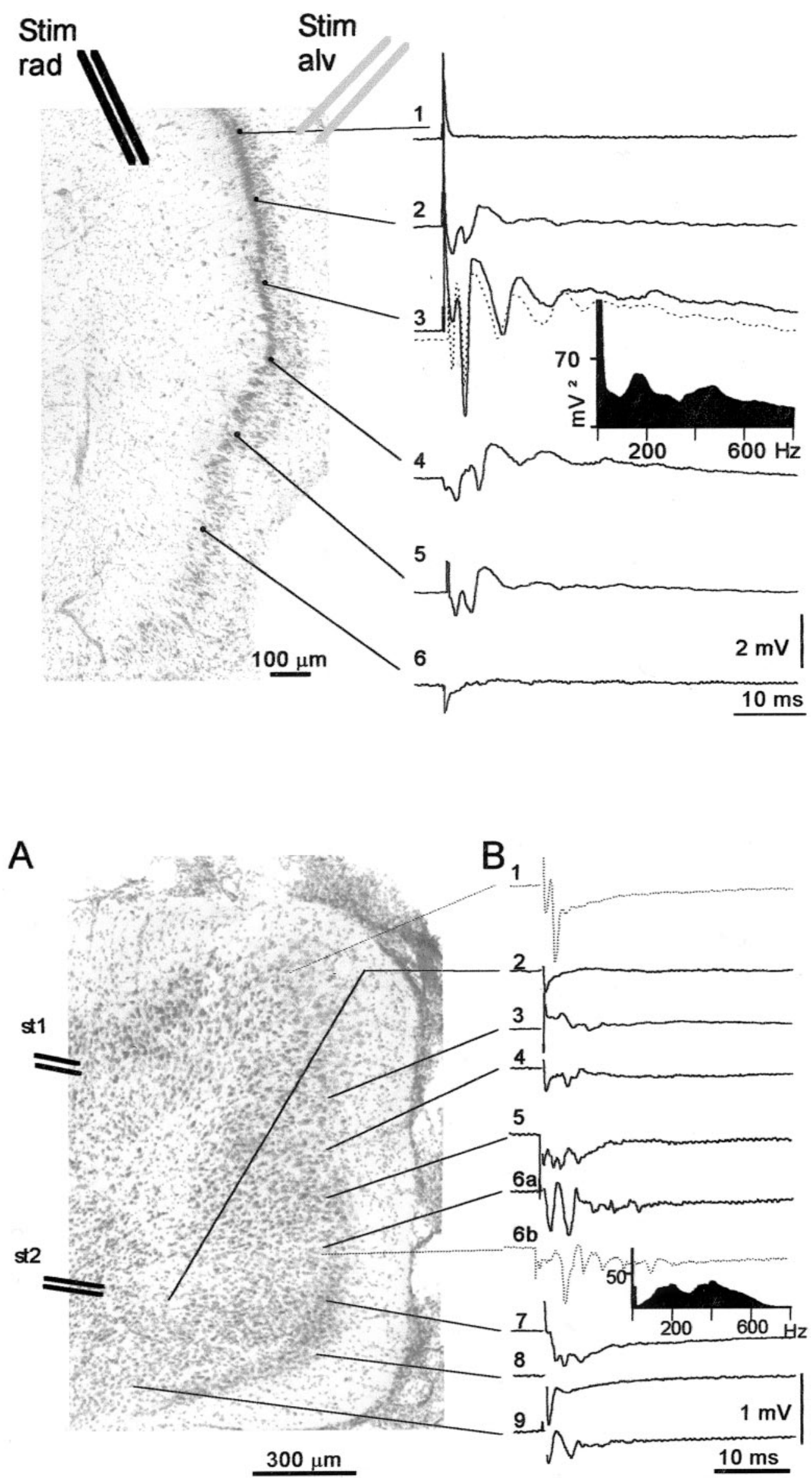

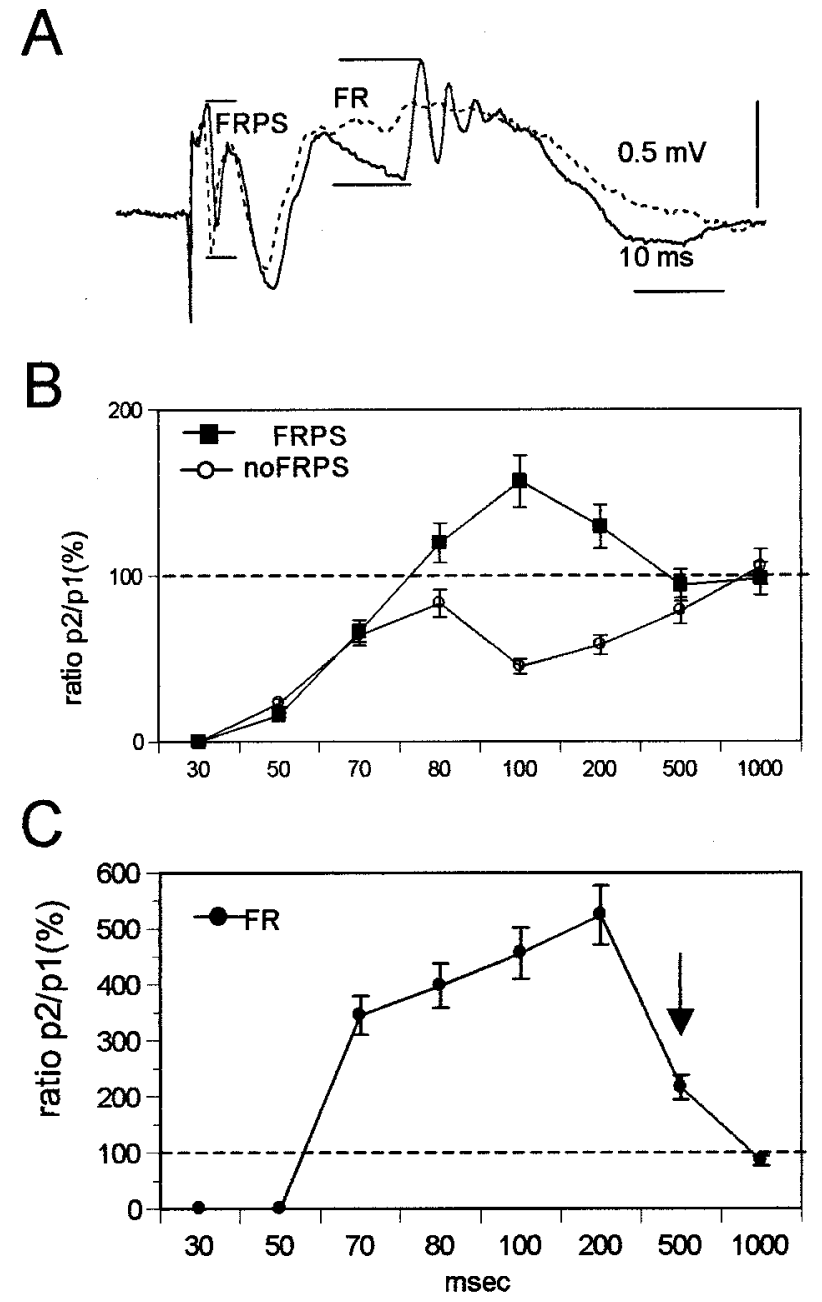

Figure 6. In vivo study of paired-pulse suppression of dentate gyrus population spikes in an epileptic rat in response to perforant path stimulation. $A$, Examples of the evoked field potentials to the conditioning (dashed line) and test (solid line) pulses delivered with an interpulse interval of $500 \mathrm{msec}$ in an area generating spontaneous FRs (FRPS). B, Paired-pulse-dependent changes at interstimulus intervals from 30 to $1000 \mathrm{msec}$ in the ratio of the population spike amplitude in response to the test stimulus (p2) to population spike amplitude in response to the conditioning stimulus $(\mathrm{p} 1) \times 100$. This ratio is plotted for responses in areas not generating FRs (noFRPS; open circles) and in areas generating FRs (filled squares). $C$, Amplitude changes in the first spike of the FR complex (filled circles) at increasing interpulse intervals. Note that FR oscillations become augmented in response to the test pulse at interstimulus intervals of $70-500 \mathrm{msec}$.

\section{Stimulation-evoked fast ripples in vitro}

During in vitro paired-pulse experiments, clear FR-like population bursts were evoked by both conditioning and test pulses. Figure $7 A$ shows an evoked DG response to hilar conditioning pulse (solid line) and test pulse (dotted line) at a $300 \mathrm{msec}$ interval. Both augmentation (as shown in $7 A$ ) or suppression (data not shown) were observed, depending on the interval between pulses. When suppression of the test response occurred, the number of population spikes in the burst would decrease, with later population spikes disappearing first until only the first population spike remained. In Figure $7 C$, paired-pulse testing showed suppression of the number of population spikes in a population burst during interpulse intervals of $15-500 \mathrm{msec}$. A similar extended period of suppression of population spike components occurred during

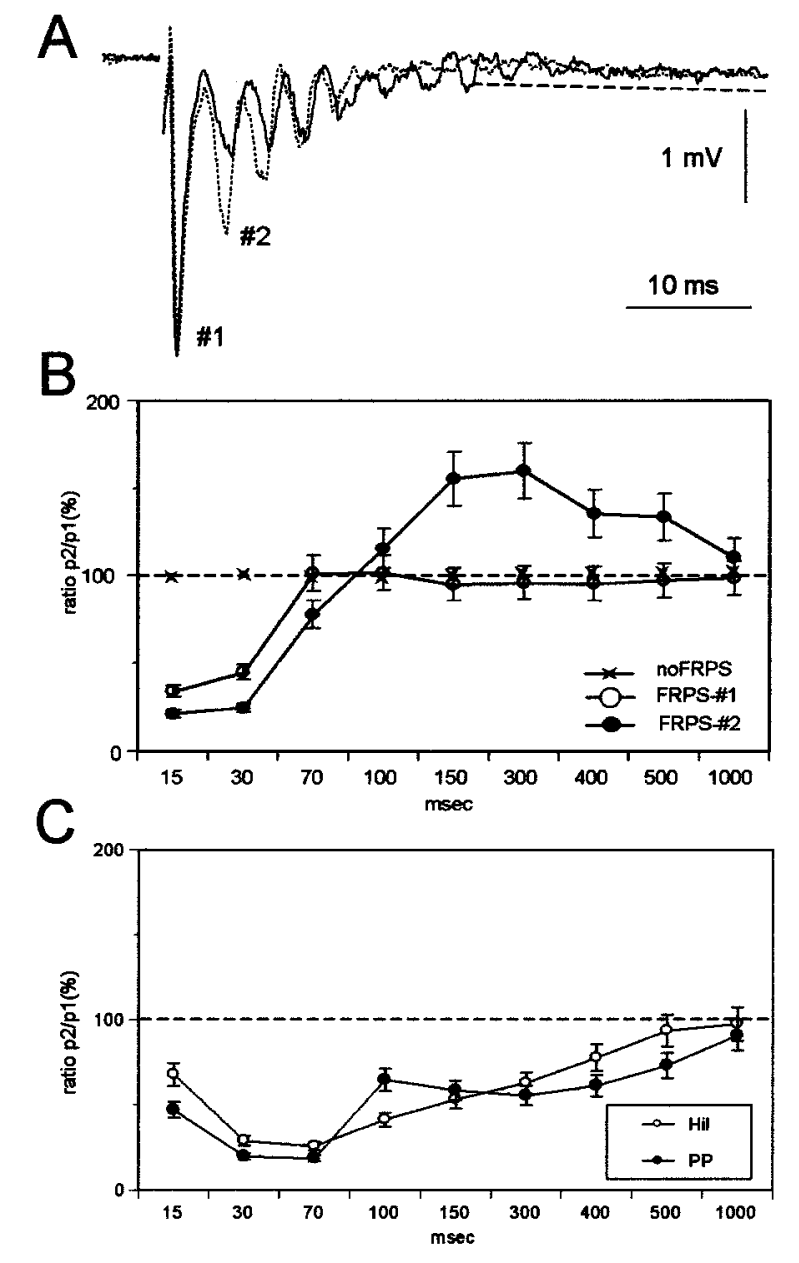

Figure 7. In vitro study of paired-pulse suppression of dentate gyrus population spikes in an epileptic rat in response to perforant path stimulation. $A$, An example of an FR-like response to hilus stimulation with conditioning pulse (solid line) and test pulse (dashed line) delivered with $300 \mathrm{msec}$ interval. \#1 and \#2 indicate the first and second spike in the response that were used for measurement of change in amplitude at different interpulse intervals. $B$, The progression of the first (open circles) and second (filled circles) spikes of FR-like responses at different interpulse intervals. noFRPS, Areas not generating FRs; FRPS, areas generating spontaneous FRs. $C$, The evolution of the number of spikes in the FR-like response to perforant path (PP; filled circles) and hilus (Hil; open circles) stimulation at different interpulse intervals.

perforant path stimulation. Figure $7 B$ illustrates that the amplitude of the population spike in an area not generating FRs but only a single population spike shows no change at conditioningtest pulse intervals of 15 to $1000 \mathrm{msec}$ ( $x$ on dashed baseline). In an area generating FR-like responses (Fig. $7 B$ ), the amplitude of the first population spike of the burst (Fig. $7 A, \# 1$ ) was suppressed by preceding conditioning pulses at 15 and $30 \mathrm{msec}$ intervals but remained unaltered at longer intervals (open circles). The amplitude of the second population spike of the FR-like response (Fig. 7A, \#2) showed a similar suppression at 15 and 30 msec intervals but became augmented at 100-500 msec interpulse intervals (Fig. 7B).

These experiments illustrate that paired-pulse suppression or facilitation is present in areas of FR-like responses. For the first two components of the hilar responses in DG, the results of paired-pulse testing were dependent on whether or not the test stimulation evoked an FR-like response in the recorded DG area. 


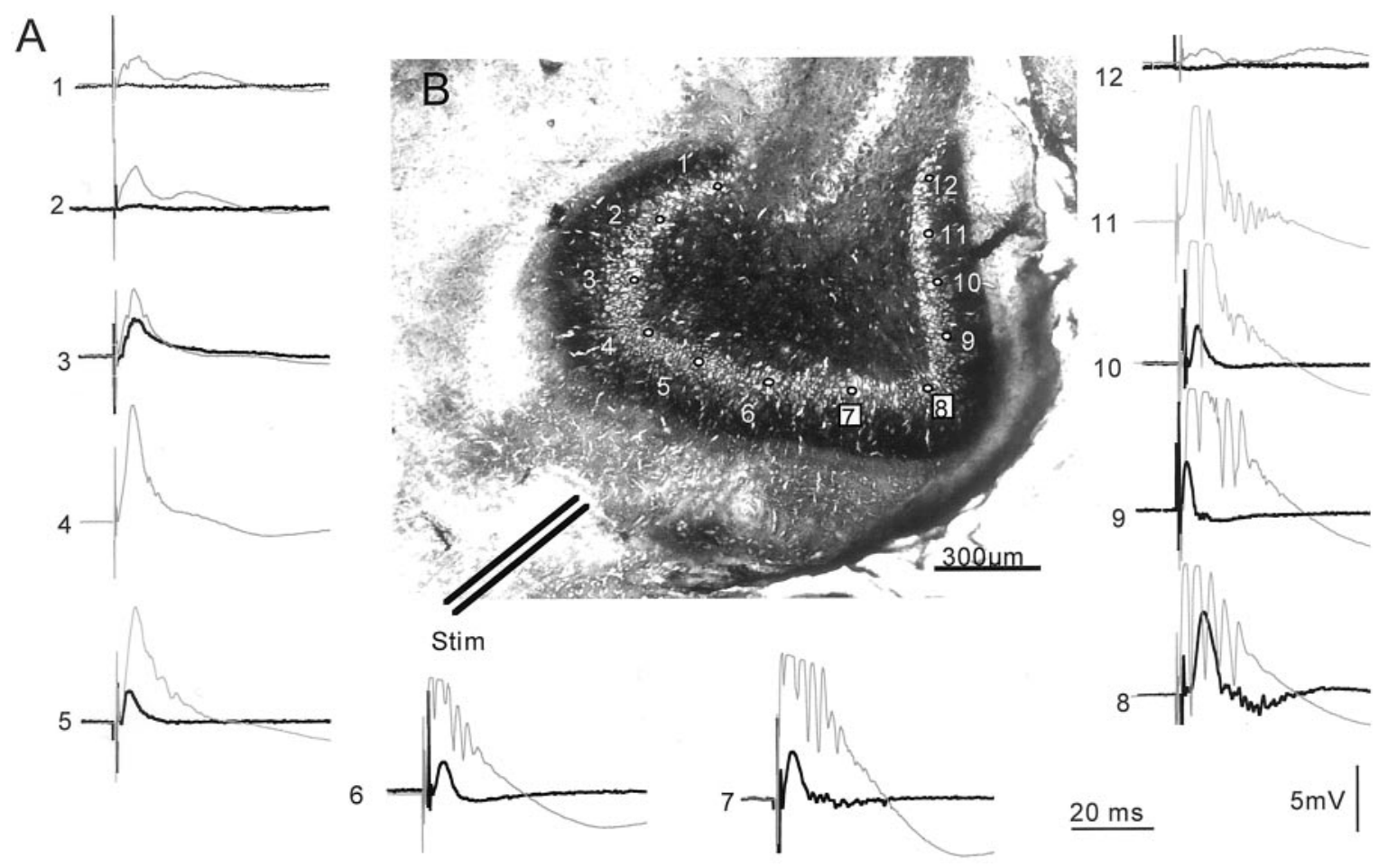

Figure 8. A, Areas in the in vitro dentate gyrus generating FR-like responses in normal ACSF (thick lines) and after the addition of $10 \mu \mathrm{M}$ bicuculline to the perfusate (thin lines). B. Timm's-stained section demonstrating sprouting of mossy fibers in the inner molecular layer in relation to the positions of the recording sites that were stimulated to evoke the field potentials shown in $A$.

Although increasing the concentration of extracellular potassium up to $5 \mathrm{~mm}$ did not significantly change the size of the area generating FR-like responses, adding the $\mathrm{GABA}_{\mathrm{A}}$ receptor antagonist bicuculline $(10 \mu \mathrm{M})$ to this media produced a substantial enlargement of the area of evoked FR generation (Fig. 8). Spontaneous FR-like bursts with a pattern similar to evoked FR were also observed during bicuculline administration (data not shown).

\section{Interaction between clusters in DG- and EC-generating FRs in vivo}

As described above, FRs recorded with several microelectrodes in the DG and EC (Fig. 1) may occur simultaneously in all recording areas or locally in only some of the microelectrodes. Crosscorrelation analysis of FRs recorded in the DG and EC revealed that some points generating FRs are closely related to each other, and some had only a weak association. Figure 9 illustrates autocorrelograms and cross-correlograms of FRs in the DG and EC recorded in vivo with fixed microelectrodes. Recordings from two microelectrode tips $1.5 \mathrm{~mm}$ apart were made in each brain region. Auto-correlograms for the activity recorded from each microelectrode are labeled with the microelectrode name (in boxes divided by vertical lines) and show prominent $400 \mathrm{~Hz}$ oscillations in each recorded area. Cross-correlograms labeled with each pair of microelectrodes indicate a positive correlation of both of the EC microelectrodes with each other and both of the EC microelectrodes with DG2. In contrast, there is an absence of any correlation between the DG1 microelectrode and any other recording site. A phase shift of $110^{\circ}$ was found between FRs in DG2 and FRs in EC1. The phase shift between FRs of DG2 and FRs of EC2 was $180^{\circ}$, and the phase shift between FRs of EC1 and EC2 was $45^{\circ}$. Perievent time histograms at longer intervals (Fig. 9C) illustrate that, although EC2 oscillations lead EC1, there is no tight unidirectional propagation of synchronous activity from EC2 to DG. Although some EC and DG oscillations are synchronous, the majority of DG1 and DG2 oscillations lead EC2 and therefore are independently generated.

FRs evoked by electrical stimulation provide an additional illustration of the close functional relationship between local areas generating FRs in the DG and EC. Figure $10 A$ shows evoked field potentials in DG and EC to pairs of electrical shocks delivered to the perforant path at $70 \mathrm{msec}$ intervals. The positions of the recording electrodes are the same as shown in Figure 9. The population spike responses to the conditioning pulse are similar in the DG1 and DG2 areas; however, FRs evoked by the test pulse occurred only in the DG2 area. These FRs are shown in the records in Figure $10 B$ containing the filtered activity in the dashed box in $A$ evoked by the test pulses. No FRs occurred in DG1. FRs recorded from the microelectrodes in both areas of EC and DG2 indicate the presence of network interactions between these three areas but not the DG1 microelectrode area.

\section{DISCUSSION}

We demonstrated the existence of local areas generating spontaneous and evoked high-frequency oscillations in the range of $200-600 \mathrm{~Hz}$, termed fast ripples, in the hippocampal entorhinal circuitry of epileptic rats. The areas generating FR appear to retain feedback inhibition in the presence of enhanced local excitatory connections. These connections may be synaptic and/or gap junctional, and additional experiments are required to elucidate the mechanisms of FR generation. The size of the area generating FRs can be on the order of $1 \mathrm{~mm}^{3}$ but can vary depending on the control of impinging inhibitory networks.

FR oscillations differ from those with a frequency range of $100-200 \mathrm{~Hz}$ in the hippocampal entorhinal circuitry of normal 
A
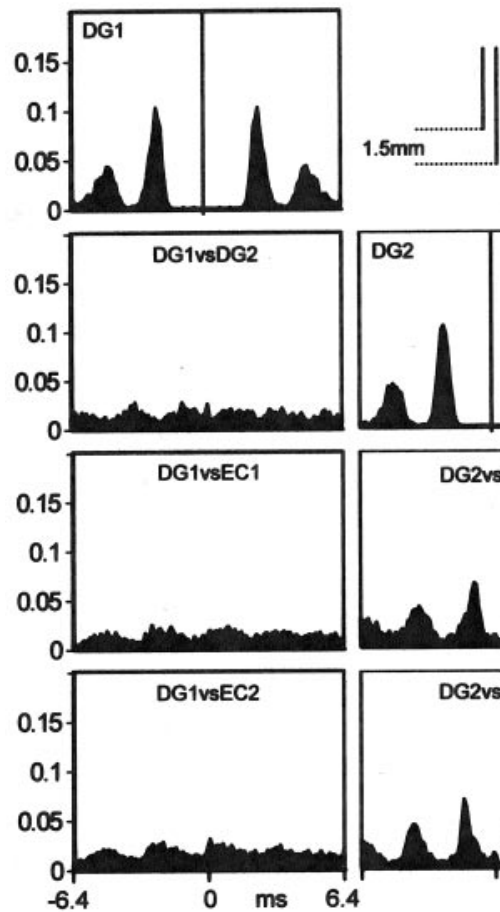

$0 \mathrm{~ms} \quad 6.4$
B
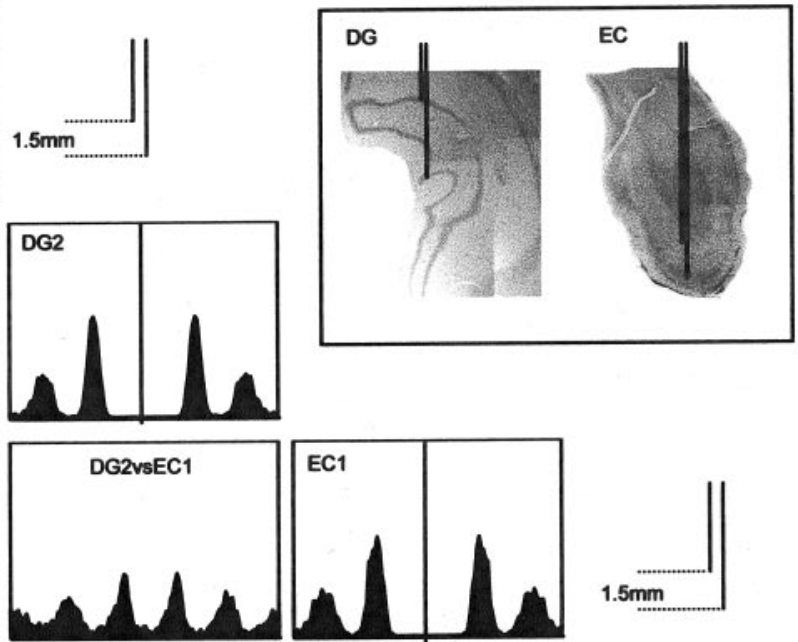

EC1

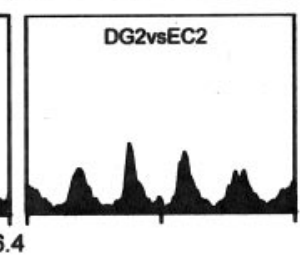

C
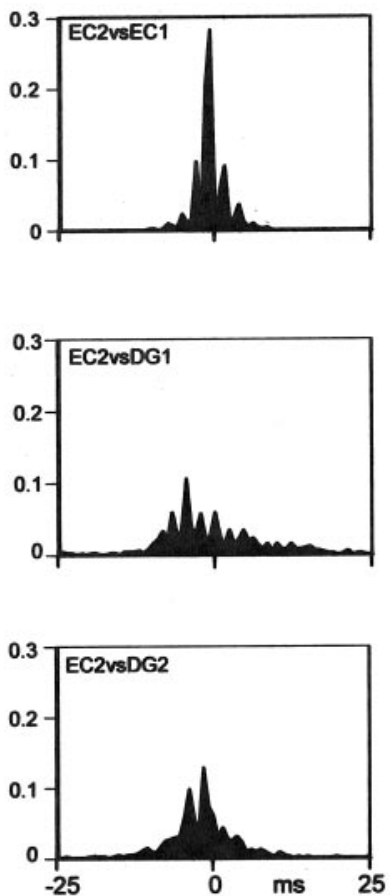

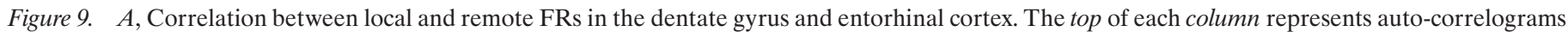

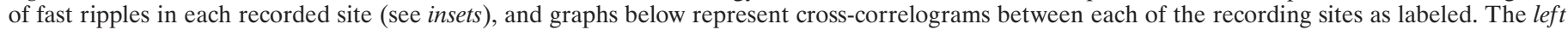

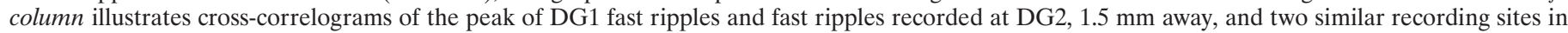

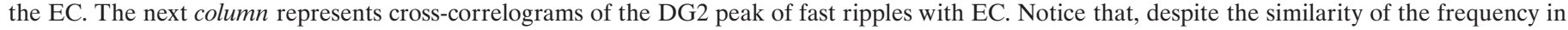

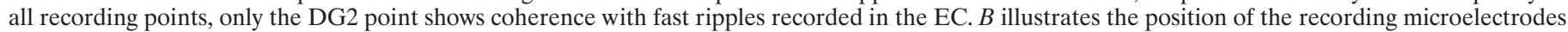

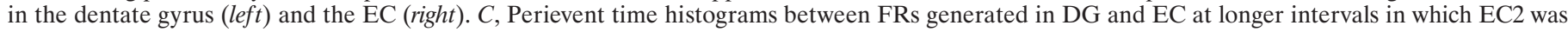
used as a trigger.

rats, which have been termed ripples (Buzsaki et al., 1992; Chrobak and Buzsaki, 1994; Ylinen et al., 1995; Chrobak and Buzsaki, 1996). In addition to the frequency difference, ripple oscillations involve a broad network of neurons (Chrobak and Buzsaki, 1996). In contrast, a local network with a higher frequency range may generate FRs. In addition, there have been no reports that ripples could be evoked by single electrical stimuli of afferents, whereas FRs can be evoked by electrical stimulation in areas in which they occur spontaneously.

According to previous studies, ripples coinciding with sharp waves appear to be a unidirectional phenomenon occurring in the CA3 area of hippocampus and propagating toward entorhinal cortex through the CA1 area, subiculum, and presubiculum (Chrobak and Buzsaki, 1996; Chrobak et al., 2000). In contrast, FRs seem to be generated in the epileptic brain locally in areas in which oscillations with such frequency do not normally occur. FRs can be synchronized between the DG and EC, and synchronization of FRs can be triggered in any of these areas (Bragin et al., 2000). The consequences of such synchronization could generate disturbances of ongoing normal brain function, which occasionally lead to seizure activity (Bragin et al., 2000).

The volume of tissue generating FRs in the DG is $\sim 1 \mathrm{~mm}^{3}$, and it may be larger in the EC. Although we did not perform a voltage versus depth profile analysis in the EC, we never observed a reversal of FRs there during recordings with a pair of microelectrodes spaced $1.5 \mathrm{~mm}$ apart, indicating that these microelec- trodes are located within the same extracellular area that generates FRs.

In the neocortex of humans and animals, oscillations in the FR range are part of the sensory-evoked response (Curio, 2000; Jones et al., 2000). In experiments searching for cellular correlates of very fast oscillations with the center frequency near $300 \mathrm{~Hz}$ in rat somatosensory cortex, no specific cell population could be found with oscillations in this frequency band, although some individual interneurons fired in synchrony with very fast oscillations (Jones et al., 2000). These fast oscillations were thought to be produced by local circuit interactions of both synaptic and gap junctional origin.

Similar mechanisms of FR generation may exist in the hippocampal-entorhinal circuitry of the epileptic brain. Simultaneous recordings of FRs in the EC and DG provide evidence for the establishment of strong functional connections between PIN clusters in different brain areas. Data presented in Figures 9 and 10 provide evidence for the existence of two PIN clusters in the DG recorded with microelectrodes separated by $1.5 \mathrm{~mm}$. One cluster of neurons generating FRs has strong functional connections with the PIN cluster in the EC. The second cluster is relatively independent, but it may have functional connections with other PIN clusters located in the EC or in unrecorded areas in the CA1-CA3 fields of hippocampus.

Another difference between high-frequency oscillations in the somatosensory cortex and hippocampal-entorhinal circuitry of epileptic rats is the range and maximum amplitude of the events. 


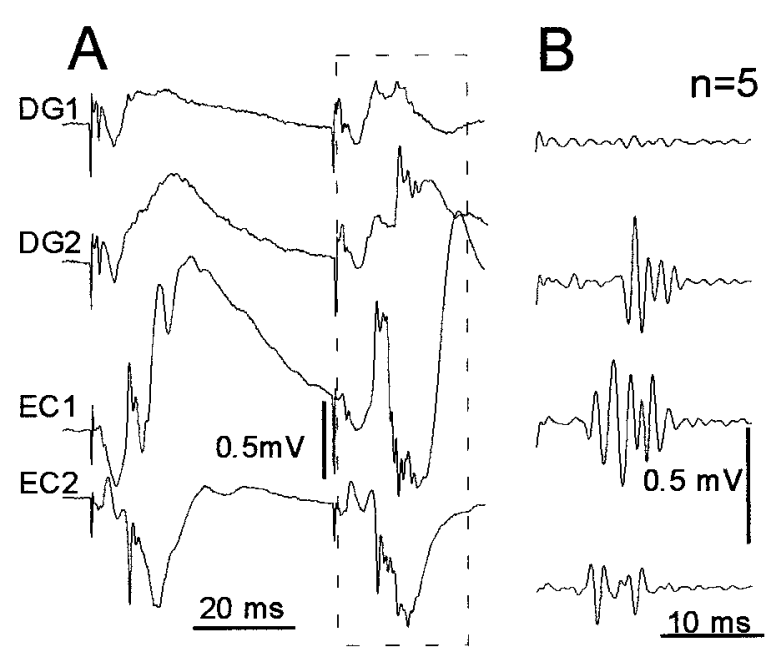

Figure 10. Occurrence of an FR-like pattern in vivo in the response to a test pulse delivered $70 \mathrm{msec}$ after the conditioning pulse $(n=5)$. The responses from the same electrodes as in Figure 8 are presented. Wide band recording $(A)$ and bandpass $(100-1000 \mathrm{~Hz})$-filtered $(B)$ examples of the responses indicated in the dashed box.

The amplitude of high-frequency oscillations of the somatosensory cortex range between 10 and $100 \mu \mathrm{V}$ (Kandel and Buzsaki, 1997; Jones et al., 2000), whereas the amplitude of ripples in the hippocampal-entorhinal circuitry range from 100 to $500 \mu \mathrm{V}$ (Buzsaki et al., 1992; Ylinen et al., 1995; Chrobak and Buzsaki, 1996). In our experiments, the FR amplitude varied between 0.1 and $1.5 \mathrm{mV}$. The amplitude of the signal depends on the distance between the recording microelectrodes and on the source of the signal and may vary significantly, but the range of the amplitude of field events gives a general indication of the number of neuronal elements participating in the generation of an electrical field event and the general relative synchrony of neuronal discharges. Mapping electrical activity with movable microelectrodes allowed us to locate areas with the highest signal. Our results suggest that synchrony of local neuronal discharges in the hippocampal-entorhinal circuitry of KA-treated rats with spontaneous seizures are much greater than the synchronous discharges of neurons in the somatosensory cortex during somatosensory-evoked responses.

Several types of morphological data are consistent with a higher density of local synaptic connections between neuronal elements in the epileptic brain attributable to neuronal death and after synaptic reorganization of the remaining neurons (Tauck and Nadler, 1985; Sutula et al., 1988; Nakajima et al., 1991). These local collaterals may be a substrate for synchronization of electrical activity leading to spontaneous bursts of population spikes, which form FR field oscillations. Our working hypothesis is that inhibition may be weak in areas generating FRs. Pairedpulse testing in our in vivo experiments indicate that the initial components of evoked responses are suppressed in areas not generating FRs for a period up to $500 \mathrm{msec}$ and augmented for a period of 100-200 msec in areas generating FRs. As a result of the extensive connections between hippocampal interneurons (Freund and Buzsaki, 1996), one might assume the strength of inhibition to be the same between areas generating FRs and those not generating FRs.

Data obtained from our in vitro experiments also support the hypothesis of increased local excitatory connections between principal cells as a possible substrate of FR generation. The number of population spikes in the bursts evoked by a second shock in the paired-pulse test were equally reduced during both perforant path and hilar stimulation at all interpulse intervals (Fig. $7 C$ ), indicating the strong inhibition in the DG of the epileptic hippocampus. The 100-200 msec period of augmentation may be attributable to activation of local excitatory connections; however, the participation of gap junctions in the generation of FRs cannot be excluded. It has been shown previously in hippocampal slices of epileptic rats (Tauck and Nadler, 1985) and has been suggested by computer simulation experiments (Traub et al., 1999) that axoaxonal gap junctions, in combination with recurrent excitatory synapses, can induce the occurrence of highfrequency population spikes superimposed on epileptiform field potentials. If interneuron properties are added to the model along with chemical synaptic interactions between respective cell types, such networks can also generate high-frequency oscillations (Traub and Bibbig, 2000).

The experiments in which the $\mathrm{GABA}_{\mathrm{A}}$ receptor blocker bicuculline widens the area of DG-generating FRs indicate that the size of this area is under the control of an inhibitory network. The robust synchrony of discharges in local areas with a strong inhibitory surround may lead to the increase in contrast between excited and inhibited neuronal elements in the hippocampus, leading to a profound imbalance between inhibition and excitation.

Application of bicuculline not only enlarges the area of evoked FR generation but also provokes the occurrence of spontaneous FRs. It seems that surround inhibition is critical in restricting areas of hypersynchronous activity because a higher concentration of potassium in our superfusion medium did not evoke epileptiform discharges in control slices or in epileptic slices in areas remote from the KA lesion. Overall, our data indicate that, although inhibition is prominent in areas in which FRs are generated, the hypothesized clusters of highly interconnected excitatory neurons are capable of overcoming the interneuronal feedback inhibition resulting in generation of epileptiform bursts and may cause functional alterations in target areas leading to the formation of a distributed epileptic network supporting the spread of epileptic activity (Bragin et al., 2000).

\section{Neuronal substrate of FRs}

One possible mechanism could be that FRs represent a field of synchronous action potentials (burst of population spikes) similar to epileptiform bursts described in numerous experiments on acute models of epilepsy. An alternative hypothesis of FR generation is that FRs may reflect inhibitory PSPs of principal neurons occurring as a result of synchronous discharges of the interneuronal network. This hypothesis was proposed for generation of oscillations with ripple frequency (Ylinen et al., 1995). Our in vitro data showing increased amplitude and spatial distribution of FRs in the presence of bicuculline support the first hypothesis that spontaneous and evoked FRs may be a field event reflecting action potentials of both principal cells and interneurons.

One interesting observation in our in vivo experiments is that FR oscillations in response to perforant path stimulation did not occur as part of the first fEPSP but were triggered by the second fEPSP at 10-30 msec latency (Figs. 6 $A, 10$ ). According to some data (Buzsaki et al., 1990; Wu et al., 1998), the late components of the evoked potentials in hippocampus reflect activation attributable to signals coming from EC after reverberation along polysynaptic hippocampal-entorhinal cortex circuitry. After a single electrical shock, an initial excitation spreads through the hippocampal-entorhinal circuitry and is amplified so that, during 
the second component of the response, recruitment into synchronous discharges of increasing numbers of the neuronal elements occur, subsequently reflected in the occurrence of FR oscillatory activity. FR generation may thus indicate the breakdown of the EC-DG gate function.

In conclusion, our experiments provide insight into the spread of pathological epileptic activity between different brain areas, supporting the generation of seizure activity despite strong inhibition. We presented evidence in epileptic brain for the existence of clusters of neurons with strong local excitatory connections. Despite strong inhibitory control of excitability by an interneuronal network, local excitatory connections within PIN clusters are capable of overcoming this inhibition and involve discharges of similar clusters in target brain areas, ultimately leading to seizures.

\section{REFERENCES}

Bertram EH, Zhang DX, Mangan P, Fountain N, Rempe D (1998) Functional anatomy of limbic epilepsy: a proposal for central synchronization of a diffusely hyperexcitable network. Epilepsy Res 32:194-205.

Bragin A, Jando G, Nadasdy Z, Hetke J, Wise K, Buzsaki G (1995) Gamma $(40-100 \mathrm{~Hz})$ oscillation in the hippocampus of the behaving rat. J Neurosci 15:47-60.

Bragin A, Engel Jr J, Wilson CL, Fried I, Mathern GW (1999a) Hippocampal and entorhinal cortex high-frequency oscillations (100-500 $\mathrm{Hz}$ ) in human epileptic brain and in kainic acid-treated rats with chronic seizures. Epilepsia 40:127-137.

Bragin A, Engel Jr J, Wilson CL, Fried I, Buzsaki G (1999b) Highfrequency oscillations in human brain. Hippocampus 9:137-142.

Bragin A, Wilson CL, Engel Jr J (2000) Chronic epileptogenesis requires development of a network of pathologically interconnected neuron clusters: a hypothesis. Epilepsia 41:S144-S152.

Buzsaki G, Chen LS, Gage FH (1990) Spatial organization of physiological activity in the hippocampal region: relevance to memory formation. Prog Brain Res 83:257-268.

Buzsaki G, Horvath Z, Urioste R, Hetke J, Wise K (1992) High frequency network oscillation in the hippocampus. Science 256:1025-1027.

Chrobak JJ, Buzsaki G (1994) Selective activation of deep layer (V-VI) retrohippocampal cortical neurons during hippocampal sharp waves in the behaving rat. J Neurosci 14:6160-6170.

Chrobak JJ, Buzsaki G (1996) High-frequency oscillations in the output networks of the hippocampal-entorhinal axis of the freely behaving rat. J Neurosci 16:3056-3066.

Chrobak JJ, Lorincz A, Buzsaki G (2000) Physiological patterns in the hippocampo-entorhinal cortex system. Hippocampus 10:457-465.

Curio G (2000) Linking 600-Hz "spikelike" EEG/MEG wavelets ("sigma-bursts") to cellular substrates: concepts and caveats. J Clin Neurophysiol 17:377-396.
Draguhn A, Traub RD, Schmitz D, Jefferys JG (1998) Electrical coupling underlies high-frequency oscillations in the hippocampus in vitro. Nature 394:189-192.

Fisher R, Webber W, Lesser R, Arroyo S, Uematsu S (1992) High frequency EEG activity at the start of seizures. J Clin Neurophysiol 9:441-448.

Freund TF, Buzsaki G (1996) Interneurons of the hippocampus. Hippocampus $6: 347-470$.

Grenier F, Timofeev I, Steriade M (2001) Focal synchronization of ripples $(80-200 \mathrm{~Hz})$ in neocortex and their neuronal correlates. J Neurophysiol 86:1884-1898.

Jones MS, MacDonald KD, Choi B, Dudek FE, Barth DS (2000) Intracellular correlates of fast $(>200 \mathrm{~Hz})$ electrical oscillations in rat somatosensory cortex. J Neurophysiol 84:1505-1518.

Kandel A, Buzsaki G (1997) Cellular-synaptic generation of sleep spindles, spike-and-wave discharges, and evoked thalamocortical responses in the neocortex of the rat. J Neurosci 17:6783-6797.

Lomo T (1971) Patterns of activation in a monosynaptic cortical pathway: the perforant path input to the dentate area of the hippocampal formation. Exp Brain Res 12:18-45.

Mathern G, Pretorius J, Babb T (1995) Quantified patterns of mossy fiber sprouting and neuron densities in hippocampal and lesional seizures. J Neurosurg 82:211-219.

Nakajima S, Frank J, Bilkey D, Schwartzkroin P (1991) Local circuit synaptic interactions between CA1 pyramidal cells and interneurons in the kainate-lesioned hyperexcitable hippocampus. Hippocampus $1: 67-78$.

Racine RJ (1972) Modification of seizure activity by electrical stimulation. I. After-discharge threshold. Electroencephalogr Clin Neurophysiol 32:269-279.

Sutula T, He XX, Cavazos J, Scott G (1988) Synaptic reorganization in the hippocampus induced by abnormal functional activity. Science 239:1147-1150.

Tauck D, Nadler J (1985) Evidence of functional mossy fiber sprouting in hippocampal formation of kainic acid-treated rats. J Neurosci 5:1016-1022.

Traub RD, Bibbig A (2000) A model of high-frequency ripples in the hippocampus based on synaptic coupling plus axon-axon gap junctions between pyramidal neurons. J Neurosci 20:2086-2093.

Traub RD, Schmitz D, Jefferys JG, Draguhn A (1999) High-frequency population oscillations are predicted to occur in hippocampal pyramidal neuronal networks interconnected by axoaxonal gap junctions. Neuroscience 92:407-426.

Traub RD, Whittington MA, Buhl EH, LeBeau FE, Bibbig A, Boyd S, Cross H, Baldeweg $\mathrm{T}$ (2001) A possible role for gap junctions in generation of very fast EEG oscillations preceding the onset of, and perhaps initiating, seizures. Epilepsia 42:153-170.

Wu K, Canning KJ, Leung LS (1998) Functional interconnections between CA3 and the dentate gyrus revealed by current source density analysis. Hippocampus 8:217-230.

Ylinen A, Bragin A, Nadasdy Z, Jando G, Szabo I, Sik A, Buzsaki G (1995) Sharp wave-associated high-frequency oscillation $(200 \mathrm{~Hz})$ in the intact hippocampus: network and intracellular mechanisms. J Neurosci 15:30-46. 